\title{
Role of Fluoroethyl Tyrosine Positron Emission Tomography FET-PET-Computed Tomography CT Scan in Differentiating Ewing's Sarcoma from Osteomyelitis
}

\author{
Nayab Mustansar* \\ Department of Nuclear Medicine, Women Medical College, Pakistan \\ *Corresponding Author: Nayab Mustansar, Department of Nuclear Medicine, Women Medical College, Pakistan.
}

Received: September 17, 2019; Published: October 14, 2019

DOI: $10.31080 /$ ASPS.2019.03.0417

\begin{abstract}
Ewing sarcoma is undifferentiated sarcoma. For confirmation of Ewing sarcoma cytological confirmation is needed and hence is very difficult to differentiate between Ewing sarcoma and Osteomyelitis via CT scan and X-ray. FDG PET is very nonspecific tracer in terms of differentiation between malignant or inflammatory lesions but in case of Ewing sarcoma, it shows increased LAT1 transporter expression which was utilized specifically to target the tumour cells and differentiate them from inflammatory lesion. So, FET positron emission tomography-computed tomography can differentiate infective pathology from malignancy and a useful tool in monitoring response to therapy.
\end{abstract}

Keywords: Ewing's Sarcoma; Flouro-Ethyl Tyrosine; Osteomyelitis

\section{History}

A 20-year-old male having Ewing's sarcoma in the region of Right humerus, who had undergone surgery and was receiving radiotherapy was referred for the evaluation of recurrence at our hospital. The presenting complaint of the boy at that was a swelling in the vault of skull that was palpable and a sinus at right humours which was a discharging sinus, which was exactly present at the site of irradiation.

Investigations

An 18F-FDG PET-CT study was performed 60 min after intravenous injection of $370 \mathrm{MBq} 18 \mathrm{~F}-\mathrm{FDG}$, after 6-h fasting with a wholebody full-ring PET-CT camera. The CT portion was performed, and subsequently PET images were taken according to the protocol.

Later on the attenuation correction was done and fusion images were formed for localization of lesion. Finally, digital images were interpreted using Xeleris ${ }^{\mathrm{TM}}$ workstation.

An FDG-avid lytic-sclerotic lesion with destructive pattern was seen in the mid-humerus and distal humerus. Moreover, there was evidence of a poorly defined lytic lesion frontoparietal region of the skull vault with increased FDG uptake.

The patient was called again after 2 days for FET PET/CT scan.

FET PET-CT showed no evidence of uptake of tracer in the humerus, but there was an evidence of increased uptake in the skull lesion.

Cytopathological reports from both lesions showed that skull lesion is a recurrence of small- round-cell tumor, whereas biopsy from the right humerus reflected only inflammatory cells and chronic infectious osteomyelitis.

\section{Discussion}

FDG/PET has unprecedented utility in diagnosing, staging and evaluating response to therapy for Ewing's scarcoma but it is non specific and most of the times its scan findings regarding evaluation of response to therapy are misleading, specially in spot surgical cases where graft infection chances are very high [1]. The radiological findings of Ewing Sarcoma is similar to that of Osteo- 
myelitis [2]. This leads to uncertainty in deciding the further management plan. Mostly false negative results have been observed via fine needle aspiration cytology of Malignancy or residual tumour [3]. As FDG is non specific it shows uptake in both malignancy and infection, so differentiating between both isn't possible this way [1]. FDG has inferior capability in distinguishing neoplastic from inflammatory or treatment-related lesions as opposed to an aminoacid PET tracer [4]. A need, therefore, arises to differentiate malignant pathology from infective pathology through imaging modalities where detection through invasive cytological procedures is difficult to perform (e.g., lesions involving spinal cord) [5]. It has been found that Ewing's sarcoma has increased LAT1 transporter expression at the cell surface [6-9]. This property has been utilized to specifically target the tumor cells and differentiate them from inflammatory lesions. FDG scan shows an increased uptake in the lesion of skull and Right humerus, whereas FET uptake was noted only in the skull lesion. Thus, FET PET-CT can serve as a useful tool in diagnosing recurrence or residual Ewing's sarcoma and response to therapy.

\section{Conclusion}

FET PET/CT is a good tool in differentiating a malignant lesion from inflammatory lesion however its use in the response to therapy is still a question mark and needs further validation.

\section{Bibliography}

1. Guimarães JB., et al. "The importance of PET/CT in the evaluation of patients with Ewing tumors". Radiologia Brasileira 48 (2015): 175-180.

2. McCarville MB., et al. "Distinguishing osteomyelitis from Ewing sarcoma on radiography and MRI". American Journal of Roentgenology 205 (2015): 640-650.

3. Kilpatrick SE and Geisinger KR. "Soft tissue sarcomas: The usefulness and limitations of fine-needle aspiration biopsy". American Journal of Clinical Pathology 110 (1998): 50-68.

4. Buck D., et al. "18F-FDG PET detects inflammatory infiltrates in spinal cord experimental autoimmune encephalomyelitis lesions". The Journal of Nuclear Medicine 53 (2012): 12691276.

5. Kebir S., et al. "18F- fluoroethyl-L-tyrosine positron emission tomography-guided diagnosis of a malignant intramedullary spinal cord tumor". Oncology Letter 12 (2016): 4705-4707.
6. Kebir S., et al. "18F- fluoroethyl-L-tyrosine positron emission tomography for the differential diagnosis of tumefactive multiple sclerosis versus glioma: A case report". Oncology Letter 11 (2016): 2195-2198.

7. Lee TS., et al. "Comparison of 18F- FDG, 18F-FET and 18F-FLT for differentiation between tumor and inflammation in rats". Nuclear Medicine and Biology 36 (2009): 681-686.

8. Tsuji AB., et al. "Comparison of 2- amino-[3-11C] isobutyric acid and 2-deoxy-2-[18F] fluoro-D-glucose in nude mice with xenografted tumors and acute inflammation". Nuclear Medicine Communications 33 (2012): 1058-1064.

9. Pauleit D., et al. "18F-FET PET compared with 18F-FDG PET and CT in patients with head and neck cancer". The Journal of Nuclear Medicine 47 (2006): 256-261.

\section{Volume 2 Issue 11 November 2019 (C) All rights are reserved by Nayab Mustansar.}

